\title{
A simple model of fully-faceted grain growth and coarsening with non-linear growth laws
}

\author{
Catherine M. Bishop ${ }^{a, b} \quad$ Raphaelle L. Satet ${ }^{c}$ \\ Rowland M. Cannon ${ }^{d} \quad$ W. Craig Carter ${ }^{a}$ \\ Andrew R. Roosen ${ }^{e}$ \\ $a$ \\ Department of Materials Science and Engineering, Massachusetts Institute of Technology, Cambridge, MA, USA \\ $b$ \\ Department of Materials, University of Oxford, Oxford, UK \\ $c$ \\ Institute of Ceramics in Mechanical Engineering, University of Karlsruhe, Karlsruhe, Germany \\ $d$ \\ Materials Sciences Division, Lawrence Berkeley National Laboratory, Berkeley, CA, USA \\ National Institute of Standards and Technology, Gaithersburg, MD, USA
}

\begin{abstract}
A numerical simulation of the growth and coarsening of completely faceted particles in a two-dimensional closed system is described. The particles grow from a supersaturated solution with driving forces including an anisotropic Gibbs-Thompson effect. Linear and non-linear growth laws were incorporated. This allows comparison to data obtained from physical experiments of $\mathrm{Si}_{3} \mathrm{~N}_{4}$ growing from a glass matrix with additions of various rare earth $(\mathrm{La}, \mathrm{Y}, \mathrm{Lu})$ where particle growth is more or less anisotropic (depending on the particular rare earth dopant). Simulations explore the ranges of kinetic parameters for which particle shapes tend to be dictated by thermodynamic and/or kinetic anisotropy. Comparison to data taken from a series of experiments indicates that La probably has a non-linear growth effect whereas $\mathrm{Lu}$ is less so.
\end{abstract}




\section{Introduction}

A typical silicon nitride microstructure is characterized by elongated grains and intergranular glassy films (IGFs) and pockets [1]. A bi-modal distribution of elongated grains plus weakened grain boundaries to cause intergranular fracture can strongly enhance fracture toughness and strength $[2,3,4,5]$. Silicon nitride is typically densified using $\mathrm{SiO}_{2}$ plus one or more oxide additives of $\mathrm{Al}, \mathrm{Mg}, \mathrm{Y}$ or a rare earth (RE) [1]. Different $\mathrm{RE}$ additives yield differences in the IGF; thicknesses are an increasing function of RE radius [6], and ongoing work seeks to understand implied differences in adsorption and its effect on IGF properties [7,8]. In particular, grain morphologies and growth statistics differ with different additives $[9,10,11,12]$. With toughened $\mathrm{SiC}$, chemistry changes also strongly alter grain growth characteristics $[13,14,15,16]$. Similarly, for alumina or functional ceramics containing small levels of liquid, the dopant choice strongly affects the grain anisotropy and susceptibility to abnormal grain growth, AGG [17, 18, 19, 20, 21]. Mechanisms for triggering AGG, including the role of faceted grains, remain strongly controversial $[20,21,22,23,24,25,26]$. Understanding the role of additives on grain growth remains a complex undertaking. Nonetheless, achieving better comprehension could contribute to developing superior materials and methods to produce single crystals by AGG [27].

Model experiments of growing $\mathrm{Si}_{3} \mathrm{~N}_{4}$ grains in initially supersaturated $\mathrm{RE}+$ silicon-oxynitride glass-forming liquids have been conducted to gain insight into the effects of different RE elements [10, 28, 29]. Grain growth and morphology statistics were captured during annealing glasses containing low volume fractions of particles. It is not known whether the RE elements affect the interface energy, the relevant diffusivities in the liquid, or surface attachment kinetics. Moreover, extant theory to understand the implications of such changes on microstructure evolution with liquid phase sintering is inadequate.

Ostwald ripening has been treated using mean field approximations for isotropic spherical particles growing competitively from a matrix by LSW theory, which treats the self similar microstructures eventually achieved [30, 31]. In growth laws derived for vanishing volume fractions of particles, the mean radius of particles, $\bar{r}$, in the distribution is related to time, $t$, the mean radius at $t=0, \bar{r}_{0}$, and a constant, $A_{n}$, in Eq. 1.

$$
\bar{r}^{n}-\bar{r}_{0}^{n}=A_{n} t
$$


and the growth exponents, $n$, differ for diffusion control or attachment controlled kinetics. While other solutions for coarsening show $A_{n}$ is a somewhat disputed function of volume fraction, the growth exponent remains that predicted by the limiting kinetics in isotropic systems at long times [32, 33].

Silicon nitride is highly anisotropic and exhibits a partially faceted growth shape $[10,11,28]$. Nonetheless, LSW theory has often been used to interpret anisotropic Ostwald ripening studies of $\mathrm{Si}_{3} \mathrm{~N}_{4}$. However, growth exponents determined separately for the ends and sides of particles have differed which invalidates assumptions of self-similar microstructure evolution [34] and various other nonidealities are observed [35].

Kitayama et al. devised a model for anisotropic Ostwald ripening of fully faceted particles, i.e., hexagonal prisms [35]. The local chemical potential is related to the weighted mean curvature of facets. Particle coarsening proceeds by a flux at each facet following combined diffusion and linear surface attachment rules. In these calculations, the surface energies of each facet are taken to be equal, and assumptions made include quasi-steady state, mean field and conservation of particle volume from the onset. Growth statistics were derived from successive iterations of the governing equations.

In growth experiments with $\mathrm{Si}_{3} \mathrm{~N}_{4}$, grains are not isolated and are observed to grow into each other or "lock" during growth [10, 29]. Steps on grain faces and the infrequent appearance of large facets not typically observed are also detected. In addition, the model growth experiments initially occur in an inhomogeneous medium still supersaturated with solute; the observed aspect ratios of these grains depend strongly on the RE dopant used and vary during the evolution from depletion after nucleation of new particles through the particle coarsening (Ostwald ripening) stage.

Recently, there has been renewed, extensive debate about the more general origins of abnormal grain growth. An essential feature of AGG is that the larger grains must have inherently higher interface mobilities in order to grow relatively faster than the average and, thereby, to develop and maintain a bimodal size distribution [36, 37, 38].

One school of argument has been that during coarsening of dispersed, faceted particles, if the interface attachment kinetics are controlling and, in particular, are in a regime in which attachment is limited by the density of surface ledges, then large grains will inherently have the requisite large advantage in mobility $[20,21,24,25,39]$. Perhaps this may arise simply from the nonlinearity in attachment rates as a function of driving force, but a further advantage could derive if the probable ledge density is higher in 
large grains, so that the mobility increases explicitly with particle size. However, there is little quantitative modeling of actual multigrain, microstructure evolution to test such ideas or predict behavior.

Roosen and Carter have developed a simple two-dimensional model for anisotropic particle growth and coarsening in a binary, two phase system predicated on grains being fully faceted [40]. This computational model permits particle-particle interactions, non-uniform solute distribution, and step formation. It provides fast computation of the shape evolution with explicit interface velocity laws and exact solution of the diffusion fields, while simplifying growth calculations compared to phase-field or other curvature based models. Sections of smooth interface can also be approximated as having many small facets.

In this paper, we extend the Roosen and Carter model to include nonlinear growth kinetics. Different parameters are explored to gain insight about behavior, in part, to elucidate effects of different $\mathrm{RE}$ additions on grain growth in $\mathrm{Si}_{3} \mathrm{~N}_{4}$. The physical motivation and implementation of our model are described in Section 2. Sample calculations are presented and results discussed in Sections 3 and 4. We conclude by outlining further capabilities of the model and describing future studies in Section 5.

\section{Theory}

The Roosen and Carter model for particle or grain growth and coarsening is based on a linear growth law for the facets. Defining the mobility as the ratio

of velocity to local driving force, $M \equiv v / F$, constant and uniform mobilities are employed for each facet, $M\left(\hat{n}_{f}\right)$, where $\hat{n}_{f}$ is the facet orientation and corresponds to a facet that appears on the Wulff shape. The model is designed to compute the growth in an initially supersaturated matrix also containing a fixed number of preassigned particles acting as heterogeneous nuclei. Particles first grow to deplete the matrix, i.e., the particle growth stage, and then a continuous transition ensues to competitive growth or coarsening among the particles, the Ostwald ripening phase. In the next section, we first explore the implications of other growth laws with driving force dependent mobilities, $M\left(\hat{n}_{f}, F\right)$ primarily through effects in the particle coarsening regimes; then a modified growth model is described. 


\subsection{Rate-Limiting Behavior}

Classically, the two possible rate-limiting processes in particle coarsening are far-field diffusion and surface attachment. Examining the results of LSW coarsening theory in these two limiting cases gives insight into the cross-over conditions between controlling regimes [41]. In the far-field diffusion limited case, the equations governing the mean particle size as a function of time and its rate of change are

$$
\begin{aligned}
\bar{r}^{3}-\bar{r}_{0}^{3} & =\frac{A_{d} \gamma D_{d} \Omega C_{\infty} t}{k T} \\
\frac{d \bar{r}}{d t} & =\frac{A_{d} \gamma D_{d} \Omega C_{\infty}}{3 \bar{r}^{2} k T} .
\end{aligned}
$$

In the case linear surface attachment kinetics are limiting, the corresponding equations are

$$
\begin{aligned}
\bar{r}^{2}-\bar{r}_{0}^{2} & =\frac{A_{a l} \gamma K_{a l} \Omega C_{\infty} t}{k T} \\
\frac{d \bar{r}}{d t} & =\frac{A_{a l} \gamma K_{a l} \Omega C_{\infty}}{2 \bar{r} k T} .
\end{aligned}
$$

In Eqs. 2 to $5, \gamma$ is the isotropic interface energy, $D_{d}$, the diffusivity of solute in the matrix, $K_{a l}$, the linear surface-attachment rate constant which controls the surface mobility, $\Omega$, the atomic volume of solute, $C_{\infty}$, the equilibrium mole fraction of solute in the matrix, i.e., for infinite sized particles, $k$, Boltzmann's constant, $T$ is temperature, and $A_{d}$ and $A_{a l}$ are numerical constants near unity.

The condition for a cross-over in controlling regimes can be related to a characteristic mean particle size, $\bar{r}^{*}$. An equality of the rate of change of the mean sizes, Eqs. 3 and 5 , yields $\bar{r}_{1}^{*}=\left(2 A_{d} D\right) /\left(3 A_{a l} K_{a l}\right)$.

Although the simplest growth law is a linear relation between interface velocity and driving force, more realistic rate controlling processes could be non-linear. For growth of a facet based on atom attachment at ledges associated with the emergence of a single screw dislocation, the interface velocity is a parabolic function of driving force [42]. For a facet with multiple screw dislocations, the growth law falls between parabolic and linear, in 
general. ${ }^{1}$ The growth velocity of a facet controlled by 2-D island nucleation has an exponential form where the activation energy scales inversely with driving force $[43,44]$.

Here we will consider a parabolic growth law by analogy to Eq. 5. This takes the form in Eq. 6 with $K_{a p}$ and $A_{a p}$, the parabolic growth rate constant and numerical constant, respectively. The transition between linear and parabolic attachment kinetics based on equality of rates yields $\bar{r}_{2}^{*}$, Eq. 7 .

$$
\begin{array}{r}
\frac{d \bar{r}}{d t}=\frac{A_{a p} K_{a p}}{3}\left(\frac{\gamma \Omega C_{\infty}}{\bar{r} k T}\right)^{2} \\
\bar{r}_{2}^{*}=\frac{2 A_{a p} K_{a p}}{3 A_{a l} K_{a l}} \frac{\gamma \Omega C_{\infty}}{k T}
\end{array}
$$

Then, two cases arise, Fig. 1. When $\bar{r}_{1}^{*}<\bar{r}_{2}^{*}$, the kinetics are dominated by linear attachment kinetics at small particle sizes and diffusion control at larger particle sizes. When $\bar{r}_{1}^{*}>\bar{r}_{2}^{*}$, the kinetics are dominated by linear attachment at small particle sizes and the parabolic attachment mechanism at large particle sizes, having lower driving forces.

\subsection{Linear and Parabolic Growth Laws}

The Roosen and Carter model is restricted to two dimensions with polygonal (or fully-faceted) particles growing at first isolated and then competitively while depleting solute from a matrix. The particles can have anisotropic surface energies, $\gamma\left(\hat{n}_{f}\right)$, and anisotropic mobilities, $M\left(\hat{n}_{f}\right)$, which depend on the normal, $\hat{n}_{f}$, of the facet $f$. During growth, any single facet can selfdivide into three or more facets by forming one or more small steps. Thus, the shape of a particle can change dynamically, as needed, and this algorithm has been used to simulate the formation and growth of dendrites [45] and ledge formation during surface diffusion [46]. A (small) numerical lower limit to a facet size for self-division is set for computational efficiency, but it has a negligible effect on evolution.

The diffusion equation and equation for the motion of a facet are derived in terms of the chemical potential, $\mu-\mu_{o}$, and then assuming that $\partial \mu / \partial C=$ const. The reference $\mu_{o}=\mu$ is defined for equilibrium at the interface of an

\footnotetext{
${ }^{1}$ In the instance that the dislocations form a balanced group and the driving force is below a critical value, the velocity will be zero [42].
} 

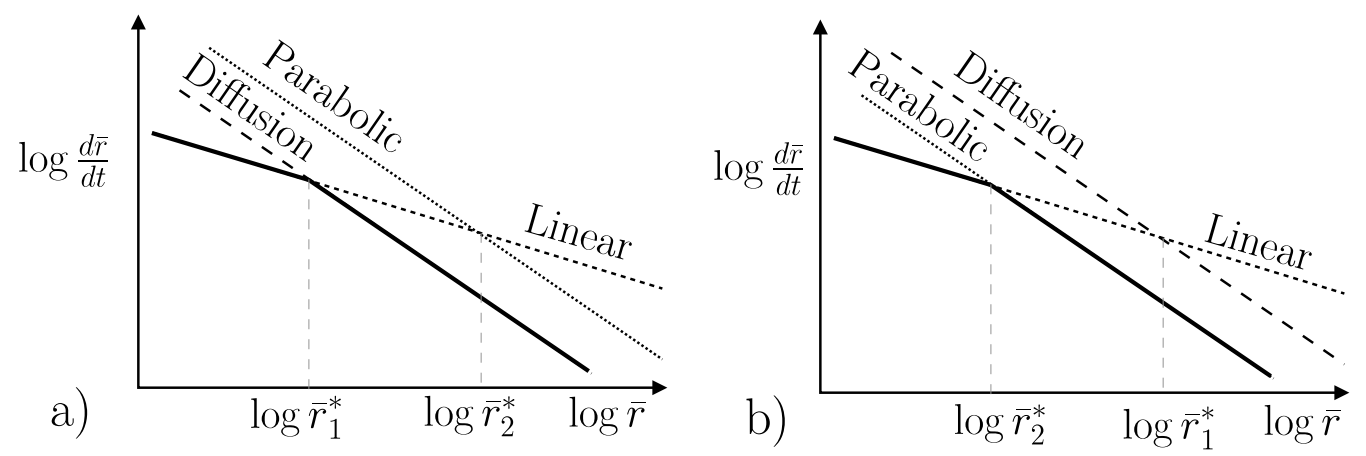

Figure 1: Schematics depict growth rates against mean particle size for diffusion control and linear and parabolic attachment control. a) Growth is limited by linear attachment at sizes below $\bar{r}_{1}^{*}$ and diffusion above. b) Growth is limited by linear attachment if below $\bar{r}_{2}^{*}$ and parabolic attachment above. As lines for parabolic attachment and diffusion-limited growth are parallel, no transition can occur between these two regimes by this measure.

infinite sized particle having an equilibrium composition $C_{\infty}$ in the matrix. It follows that for small deviations from equilibrium, the chemical potential and diffusion equations are

$$
\begin{array}{r}
\mu-\mu_{o}=\frac{\partial \mu}{\partial C}\left(C-C_{\infty}\right) \\
\frac{\partial \mu}{\partial t}=D \nabla^{2} \mu
\end{array}
$$

where $D$ is the diffusivity of solute here assumed to be constant in the matrix and very small in solid phases. The equilibrium solubility is very small in both phases. The characteristic scales for length and time are set by $L$, the size of the simulation box, and $L^{2} / D$, respectively.

The interface motion is handled as a source term in the generalized diffusion equation Eq. 10. The solute liberated at the interface by incremental motion is $\Delta C(=C)$, the interface velocity is $v$, and $\delta$ (interface) is a delta function at the interface. In the simplest case, the velocity of a facet, $v_{f}$, is a linear function of driving force, $F$, Eq. 11 meaning facet mobilities are constants. The driving force is the difference between the chemical potential in the matrix adjacent to and averaged along a facet $f,\langle\mu\rangle_{f}$, and the equi- 


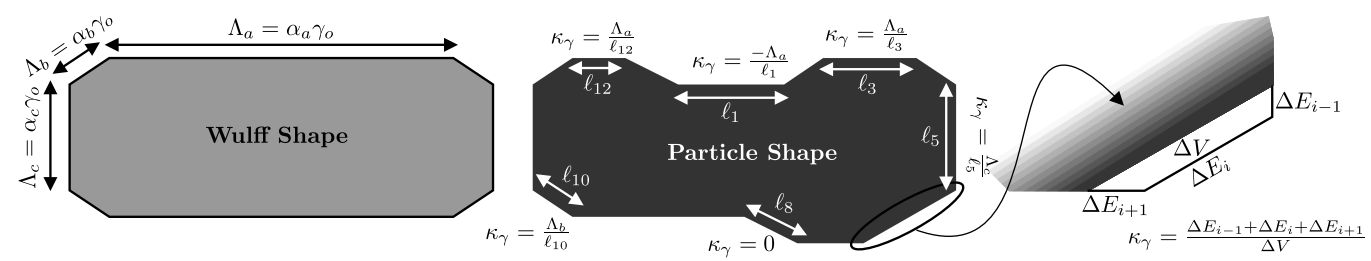

Figure 2: Illustration of weighted mean curvature, $\kappa_{\gamma}$. A Wulff shape is a construction based on the $\gamma(\hat{n})$ that gives the shape that minimizes total surface energy for an isolated map; the Wulff shape has the same physical units as $\gamma(\hat{n})$. A physical shape of a particle surface consists of the orientations that appear on the Wulff shape. The weighted mean curvature is the rate at which total surface energy is created as volume is added to the surface. For a completely faceted shape in two dimensions, a facet's weighted mean curvature is proportional to its corresponding length on the Wulff shape divided its length on the physical shape. An additional factor of \pm 1 or 0 , related to how the facet is connected to its immediate neighbors, appears because added volume may increase, decrease, or leave unchanged the surface energy.

librium chemical potential for that facet, $\mu_{e q}(f)$. The equilibrium chemical potential at a surface is affected by its geometry and surface energy $\gamma(\hat{n})$. The generalized Gibbs-Thompson effect is expressed as an increase equal to the weighted mean curvature, $\kappa_{\gamma}: \mu_{e q}(f)-\mu_{o}=\kappa_{\gamma}(f)$ [47]. A surface's local weighted mean curvature is the rate of total surface energy change with local volume addition. For isotropic surfaces $\kappa_{\gamma}=\gamma \kappa$, where $\kappa$ is the mean surface curvature. For anisotropic surfaces, the expression for $\kappa_{\gamma}$ is generally more complicated $\left(\kappa_{\gamma}=\nabla_{S} \cdot \vec{\xi}\right.$, where $\nabla_{S}$. is the surface divergence and $\vec{\xi}$ is the Cahn-Hoffman capillarity vector.) However, for completely faceted Wulff shapes (i.e., polygonal Wulff shapes), $\kappa_{\gamma}$ is not too complicated. Fig. 2 illustrates how, owing to the surface energy increase for a facet advance with its associated volume change, for a particle facet with length $\ell_{f}$ that shares the same orientation as a Wulff facet $\Lambda_{w}, \kappa_{\gamma}$ is $\sigma \Lambda_{w} / \ell_{f}$ where $\sigma$ is \pm 1 or 0 , depending on how a facet's neighbors are attached to it. Lengths in the Wulff shape have the same dimensions as surface tension; this is illustrated in Fig. 2 by scaling with a reference surface tension $\gamma_{0}$. 


$$
\begin{array}{r}
\frac{\partial \mu}{\partial t}=D \nabla^{2} \mu-v \Delta C \frac{\partial \mu}{\partial C} \delta(\text { interface }) \\
v_{f}=M\left(\hat{n}_{f}\right) F(f) \\
F(f)=\left[\langle\mu\rangle_{f}-\mu_{e q}(f)\right]
\end{array}
$$

A computational time step, $\Delta t$, entails: 1) checking whether a facet should self-divide (owing to concentration gradients parallel to itself) and recursively performing all possible self-divisions; 2) advancing the position of each facet according to Eqs. 10-12;3) updating the concentration field adjacent to each facet because of solute absorption/desorption; 4) numerically updating concentration field according to the diffusion equation. Therefore, information about the relationship between attachment kinetics and driving force is included locally in each interface velocity equation. Whether the resultant evolving microstructure is dominated by attachment or diffusion is not dictated explicitly.

In order to generalize the model to incorporate a non-linear attachment mechanism at facets of individual particles, we first note from the growth rate vs. size curves that the growth law for a facet can shift from parabolic in driving force for low positive driving forces to linear in driving force for large positive driving forces, essentially when the ledge density becomes high enough. Then, based on the definition of mobility from Section 2, for small driving force $\left(0 \leq F \leq F^{*}\right)$ the mobility of a facet can be made proportional to the driving force, $M\left(\hat{n}_{f}, F\right)=M_{0}\left(\hat{n}_{f}\right) F$, while for large driving force the mobility is kept a constant, $M\left(\hat{n}_{f}, F\right)=M_{0}\left(\hat{n}_{f}\right)$. However, here we assume linear growth laws, constant mobilities, for facets with negative driving forces as shrinking particles can dissolve beginning from corners which have infinite sources of steps.

\subsection{Modeling Silicon Nitride}

Silicon nitride crystals grown from supersaturated glass are observed to be prisms with six-sided cross sections and rounded caps at the ends, Fig. 3. One set of Satet's experimental measurements are taken on only those grains that appear rectangular in 2-D cross section and so reveal the actual grain lengths [29], from which aspect ratios can be estimated. In order to capture the essence of that experimental geometry for these 2-D calculations, silicon nitride-like microstructures and grain growth statistics will be illustrated and 


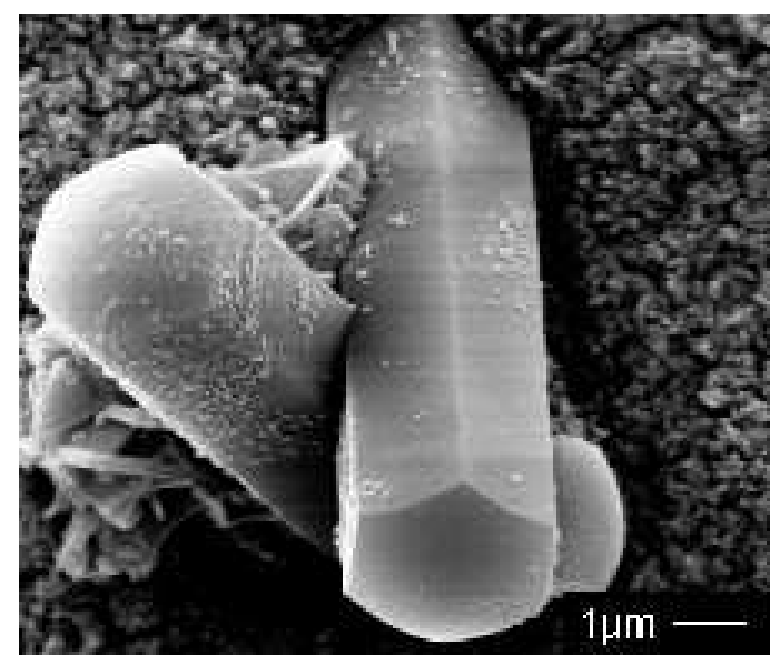

Figure 3: $\mathrm{A} \mathrm{Si}_{3} \mathrm{~N}_{4}$ grain protruding from etched oversaturated Y-Si-Mg-O-N glass (which experienced some crystallization on cooling). The grain exhibits long prism facets and rounded caps.

analyzed as derived from simulations with arrays of particles with eight facets on the Wulff shape and anisotropic mobility functions arrayed randomly in a closed 2-D box, Fig. 4. The two low energy facets represent the prism planes (sides), observed in experiment. The basal planes and rounding are captured in the inclusion of the three higher energy facets at each end. More facets could be used to give smoother ends, but many trends of interest can be captured with this simulation.

Two kinetic laws are being investigated through calculations, Fig. 5. In the first case, both end and side facets obey linear kinetic laws for all values of driving force. In the second case, all the end facets obey linear kinetic laws for all driving forces, while the sides obey a parabolic law for $0 \leq F \leq F^{*}$ and linear for all other $F$. In the former instance, the ratio of the mobilities $M_{\text {end }}(F) / M_{\text {side }}(F)$ is a constant for all driving forces. In the latter, the mobility ratio approaches $\infty$ as the driving force approaches zero, Fig. 5. In order to distinguish between kinetic and energetic dominance, here we assign distinct ratios of interface energies $\gamma_{\text {end }} / \gamma_{\text {side }}=2$ and of the high-velocity mobilities $M_{0, \text { end }} / M_{0, \text { side }}=10$.

Initial conditions are composed of a spatially uniform distribution of (nontouching) small particles in a supersaturated matrix. The particle distribu- 


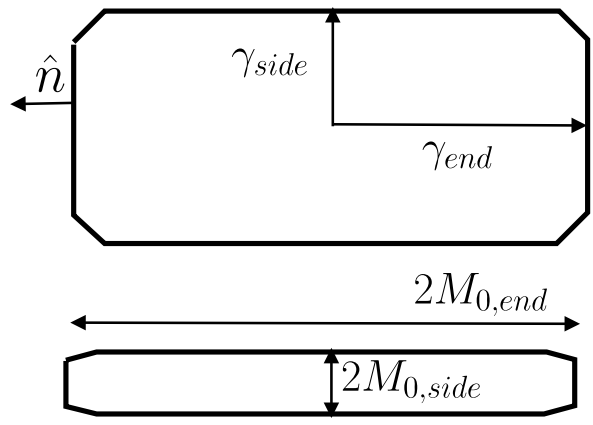

Figure 4: Depictions of the surface energy, $\gamma(\hat{n})$, and mobility constant, $M_{0}(\hat{n})$, as functions of facet orientation, $\hat{n}$.

tion consists of regular octagons with random orientations and diameters randomly chosen from the range, $0.001 \leq L=W \leq 0.01$, giving a volume fraction of order 0.001 . No new particles are produced during the simulation. Zero diffusion flux boundary conditions are specified at the edges of the computational domain, taken as a square of side length $L$. Thus, this simulates a situation where all nucleation occurs as heterogeneous nucleation and at time $t=0$; whereas the initial particles ought to have their Wulff shape if they nucleate homogeneously.

The initial solute supersaturation is a parameter that can be described in terms of the final volume fraction of second phase, $V_{f}$, (as the matrix solubility is trivial) and is 0.3 for all calculations discussed in this paper. Moreover, here, we take $\partial \mu / \partial C=1$, which defines the energy scale (e.g., for chemical potentials, driving forces and the mobility transitions $F^{*}$, as energy $/ L^{3}$ and for surface energy, as energy $\left./ L^{2}\right)^{2}$. Thus, the initial condition is that $\mu_{i}=V_{f}$ (and so the maximum supersaturation possible is $\mu_{i}=1$ ). The surface energy is scaled by $\gamma_{0}=5 \times 10^{-4}$ for this paper.

\footnotetext{
${ }^{2}$ Units referring to a three dimensional calculation are presented for clarity; however, the simulation was two-dimensional and thus $L^{3} \rightarrow L^{2}$ and $L^{2} \rightarrow L$ in the simulation.
} 

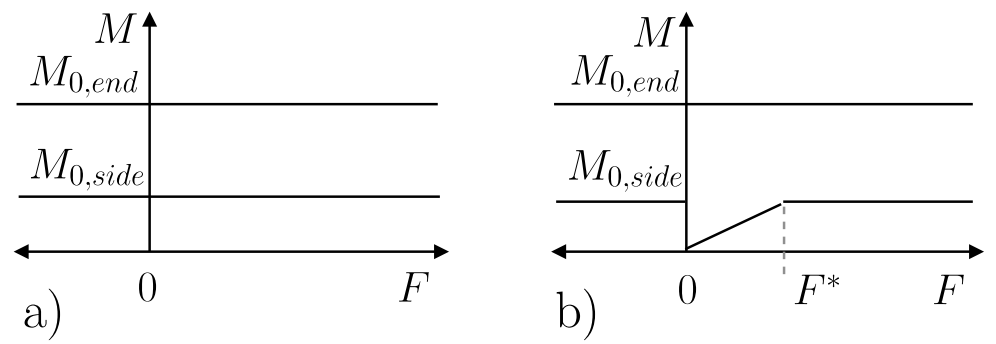

Figure 5: Schematics of the two combinations of kinetic laws being investigated. a) End and side facets each have a constant mobility for all driving forces and obey linear kinetic laws. b) The end facets follow linear kinetic laws for all $F$; the side facets obey a linear law except for $0 \leq F \leq F^{*}$ where $M_{0}(\hat{n})$, is proportional to $F$, a parabolic kinetic law.

\section{Results}

Simulations were done with arrays starting with either 40 or 100 particles per box and run until only a few particles remained. To elucidate the behavior, simulations were also run with only a single particle in the box. Typically these were done in sets with values of $D=0.5,0.05$ and 0.01 and with $F_{\text {side }}^{*}=$ $1,0.5,0.1$ and 0 and $F_{\text {end }}^{*}=0$. Two series of snapshots from multiparticle simulations are presented in Fig. 6. The first row shows images from a calculation with strictly linear kinetic laws, $F_{\text {end }}^{*}=F_{\text {side }}^{*}=0$. The second row shows images from a calculation with linear/parabolic sides and linear ends, $F_{\text {side }}^{*}=1$ and $F_{\text {end }}^{*}=0$. Owing to the slower growth law for the sides in the second case, the lengths are greater at a given time, aspect ratios (ratio of length to width) are much higher and the microstructure is more interconnected.

The maximum width, $W$, maximum length, $L$, and aspect ratio, $A=$ $L / W$, are calculated from the polygonal particle shapes. Figure 7 displays plots of the average widths, lengths and aspect ratios for the ensemble against time, on a logarithmic basis, for both single particle and 100 particle arrays, with $F_{\text {side }}^{*}$ as a parameter and $D=0.05$. In each instance, at short times, all particles are growing to deplete the matrix, the particle growth and depletion regime. ${ }^{3}$ Then, at longer times the single particles evolve toward the Wulff

\footnotetext{
${ }^{3}$ There is an exception in that some of the initial particles were, in fact, smaller than the
} 


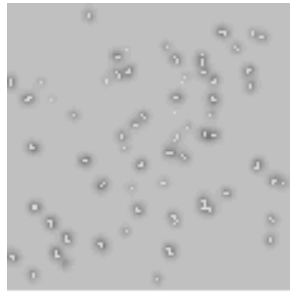

al)

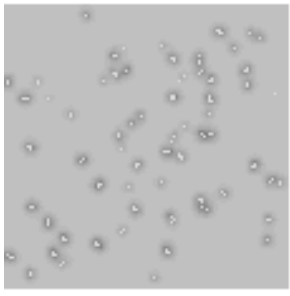

bl)

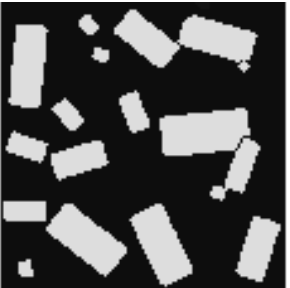

a2)

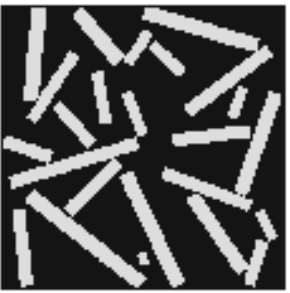

b2)

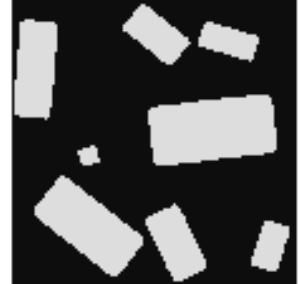

a3)

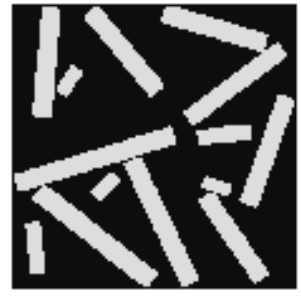

b3)

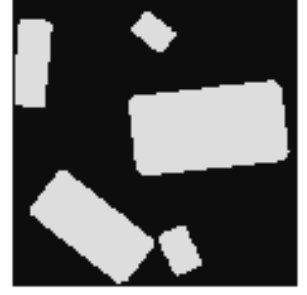

a4)

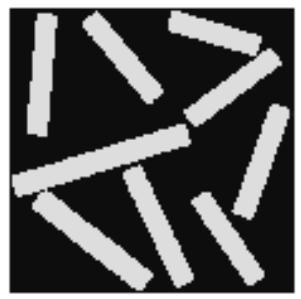

b3)

Figure 6: Corresponding images of the evolution of an initially 100 particle set with different kinetic laws. a) $F_{\text {end }}^{*}=F_{\text {side }}^{*}=0$ and b) $F_{\text {end }}^{*}=0$ and $F_{\text {side }}^{*}=1$; for both, $D=0.05$. The snapshots are taken at times $t=0.005,20,60$, and 112. The corresponding size statistics are displayed in Figs. 11 and 12. (The particles are either yellow or white and the concentration field red (dark gray) when depleted and cyan (light gray) when supersaturated. (Colors and shades may depend on observer and media.)

shape and the multiparticle arrays shift into the particle coarsening regime wherein the number of particles, $N$, begins to decrease. The array of plots in Fig. 8 illustrates the affects of $F^{*}$ and $D$ on the average aspect ratio in a set of multiparticle simulations with the same starting particle set; it tends to reach a maximum prior to the onset of the coarsening regime and then diminishes, more strongly for arrays with strictly linear kinetics. To show more detail, Fig. 9 displays the individual aspect ratios of all $N$ remaining particles versus time (during regime) over the extreme conditions of $F^{*}$ and $D$ examined.

The average aspect ratios and average lengths are plotted as a function of average width of particles in the array at several different times for

critical nucleus size, which depends on the value $\gamma_{0} / \mu_{\text {initial }}$, and so immediately dissolved; all remaining particles then grew until coarsening ensued. 
Dimensions vs. In Time for Single Particle at $\mathrm{D}=0.05$
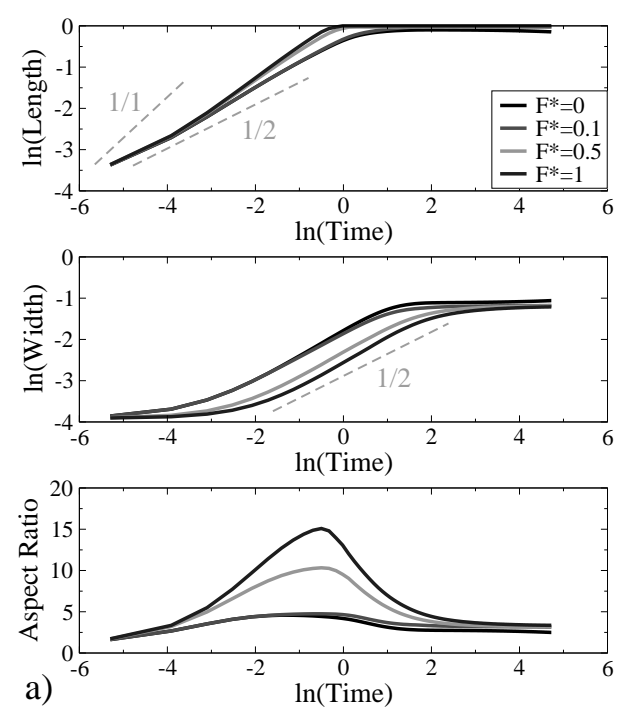

Average Dimensions vs. Time $\mathrm{D}=0.05$
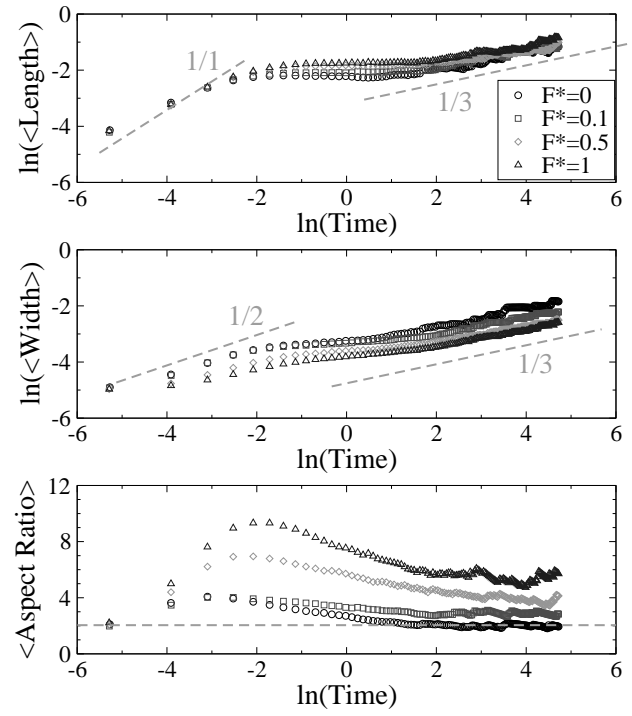

Figure 7: Plots of length, width and aspect ratio versus time for a single particle growing in a box and mean values of these for an 100 particle array with various values of $F^{*}$, all for $D=0.05$. For the initial part in the graph, particles are all growing whilst depleting the matrix; the first datum for widths is essentially the initial size. At a far longer time scale, the single particle approaches its equilibrium shape and the particle arrays begin competitive growth in which particles are disappearing. 
Average Aspect Ratios vs. Ln Time for Various D and F*

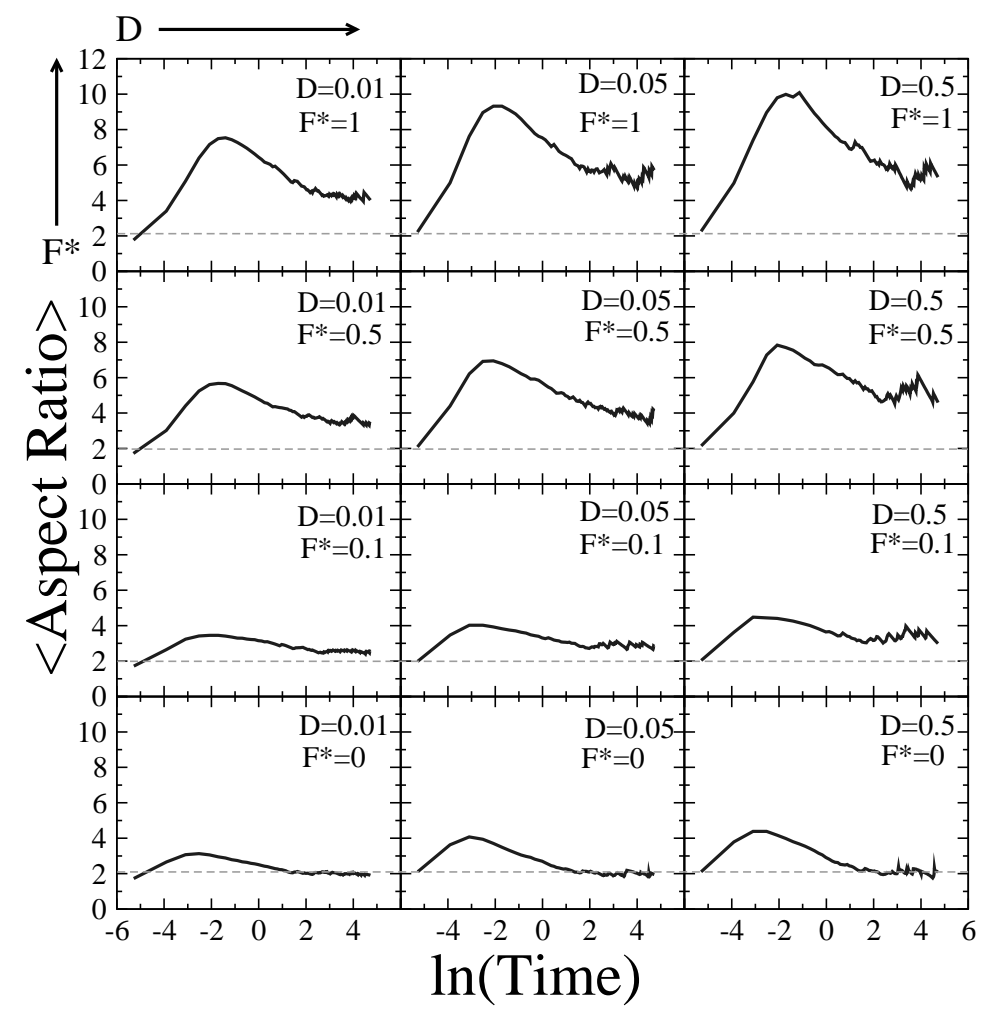

Figure 8: Array of plots showing the evolution of average aspect ratio for the range of $F^{*}$ and $D$ examined. 
Aspect Ratio Tragectories for Various $\mathrm{F}^{*}$ and D
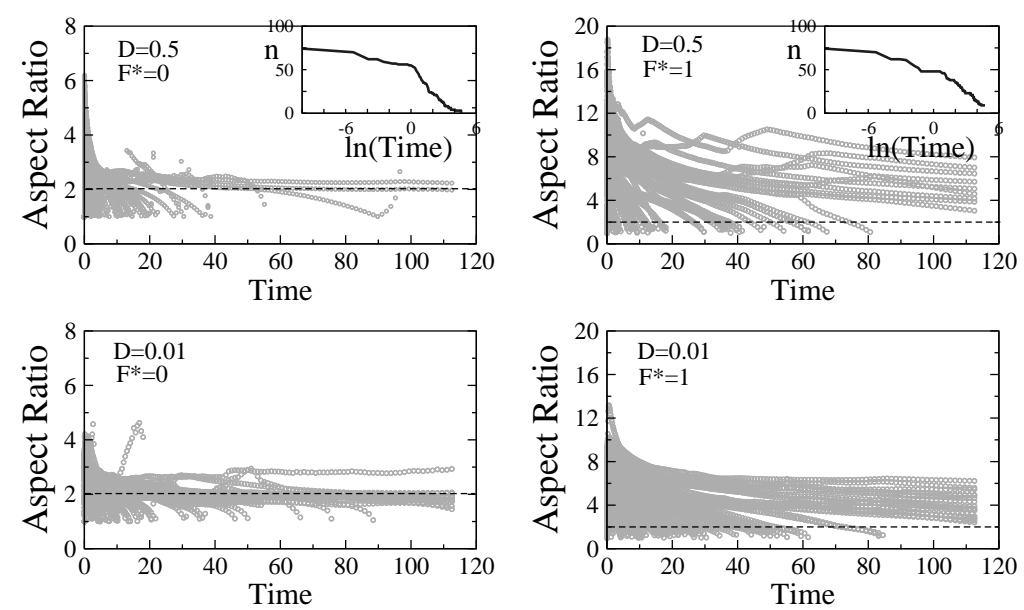

Figure 9: Set of plots showing the evolution of aspect ratios for all $N$ remaining particles versus time for the extremes of $F^{*}$ and $D$ examined. Trajectories terminate when particles disappear during coarsening. With linear kinetics, particles tend toward the Wulff shape during coarsening, until near their disappearance; whereas with nonlinear attachment kinetics, the much more anisotropic and variable shapes are dictated by kinetic anisotropy. 
Aspect Ratio and Length vs. Width for $\mathrm{F}^{*}=1,0$ and $\mathrm{D}=0.05$
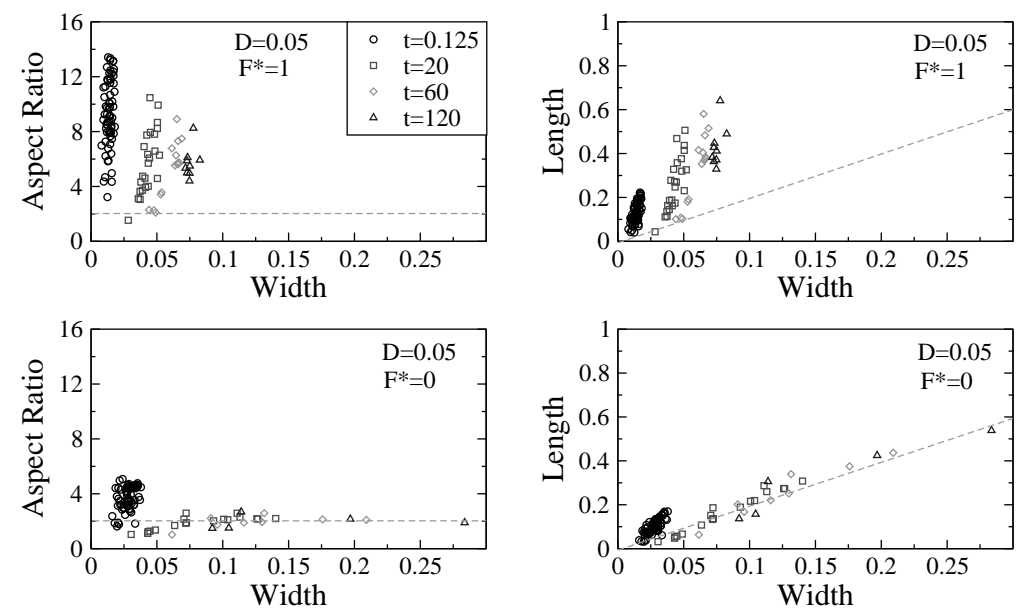

Figure 10: Plots of mean particle aspect ratio and mean length versus mean width at four times during calculations starting with same 100 particles for $D=0.05$ and $F^{*}=0$ and $F^{*}=1$.

parabolic/linear $\left(F^{*}=1\right)$ and strictly linear $\left(F^{*}=0\right)$ kinetics with $D=0.05$ in Fig. 10. Further, in order to examine the effect of varying the threshold, $F^{*}$, on the particle statistics, three non-zero values of $F^{*}$ in combination with three values of matrix diffusivity were input. The resultant mean aspect ratio and mean length vs. mean width plots are displayed in Figs. 11 and 12.

\section{Discussion}

At long times, the shape of a particle ought to converge to the Wulff shape. At short or intermediate times, the aspect ratio will reflect the kinetic laws and conditions of the facets. This guideline will be altered as the diffusivity of solute in the matrix is reduced or the attachment kinetics are highly nonlinear, $F^{*} \gg 0$. With these ideas in mind, the simulations are examined.

The snapshots from particle growth calculations in Fig. 6 illustrate the range of evolution. The nuclei start out in a supersaturated matrix which they deplete of solute as the volume fraction of particles increases, the particle growth stage, which is evident in Fig. 7, for $t \ll 0.1$. During the early stages of growth, the particles are depleting the matrix with isolated diffu- 
Aspect Ratio vs. Width for Various D and $\mathrm{F}^{*}$
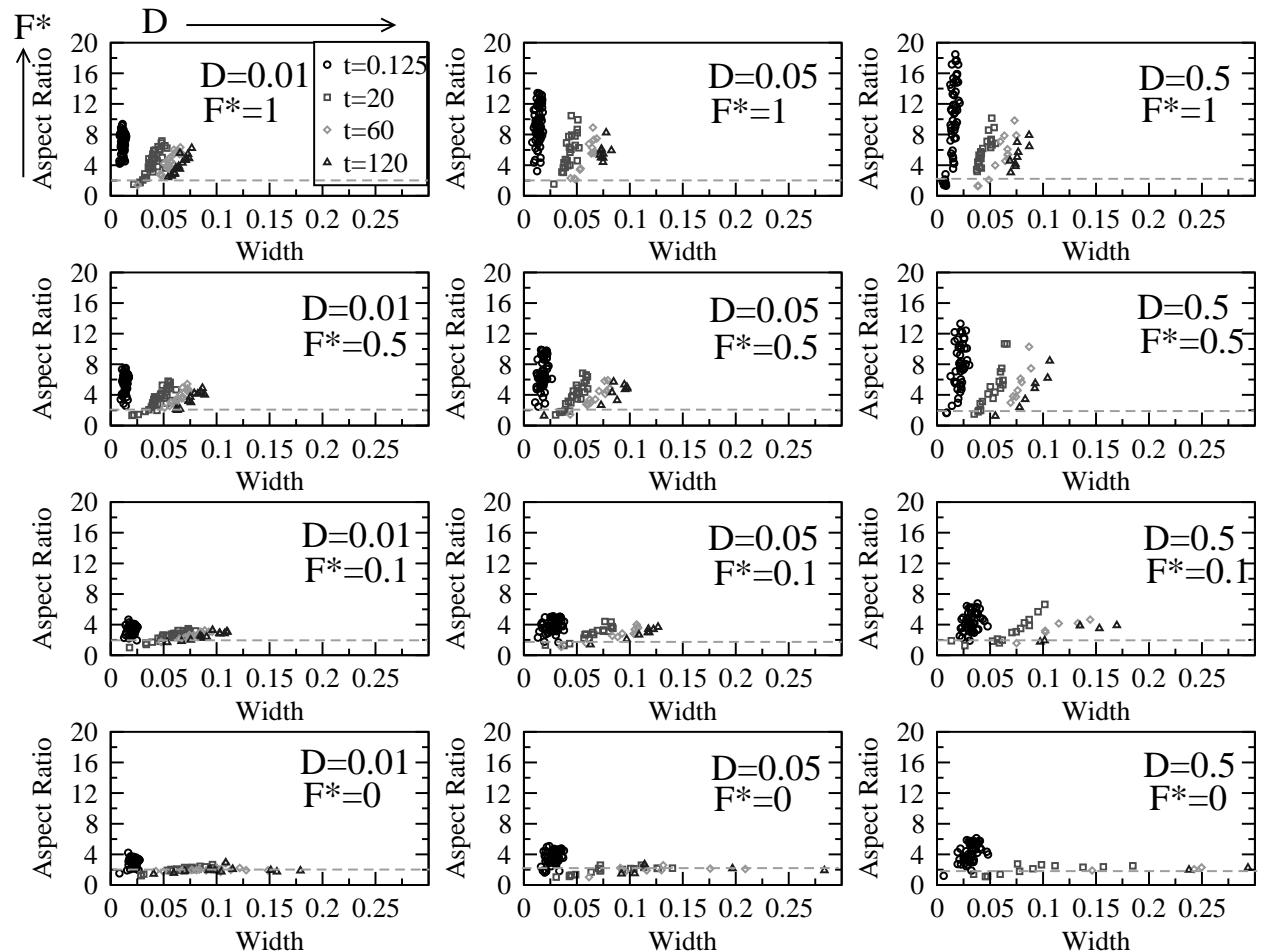

Figure 11: Plots of mean aspect ratio of particles a function of mean width at four times starting with same 100 particles for the ranges of $F^{*}$ and $D$ examined. 


\section{Length vs. Width for Various D and $\mathrm{F}^{*}$}
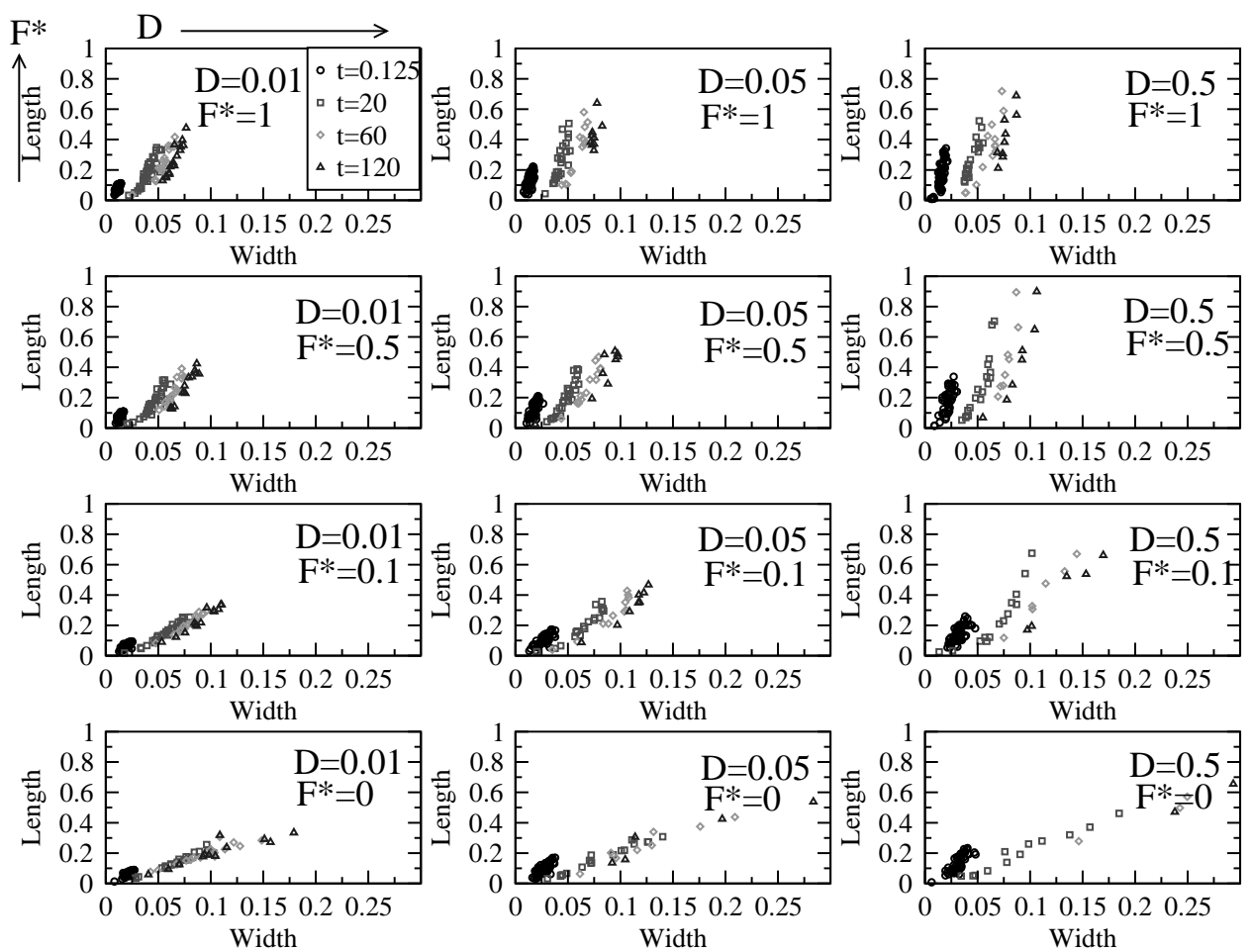

Figure 12: Plots of mean length as a function of mean width of particles at four times for the ranges of $F^{*}$ and $D$ examined, corresponding to those displayed in Fig. 11. 
sion fields. At later times, $t>10$, the growth becomes competitive at a constant particle volume fraction, the coarsening stage. (When the color of the matrix is uniform and red (or dark gray, depending how Fig. 6 is viewed), the volume fraction of particles has reached the equilibrium value.) In Fig. 6 series a), particles have strictly linear growth laws for both ends and sides. These particles coarsen with an aspect ratio close to 2 , that for the Wulff shape, as confirmed in Fig. 8. In Fig. 6 series b), the particle sides have a parabolic/linear growth rule while the ends are linear for all driving forces. In this case, the initial growth occurs at very high aspect ratio; at longer times the aspect ratios decrease but remain well above 2, Fig. 8 and are highly variable, Fig. 9. The higher interconnectivity of the particle microstructure in series 6b) is a consequence of the higher aspect ratios. This impingement limits the maximum aspect ratios, as compared with those achieved with isolated particles; these are attained toward the end of the growth stage, as is clearer in Fig. 8.

From the plots of time evolution, Fig. 7, several trends warrant comment. At the end, only a few particles remain and so the statistics show considerable fluctuations. As the time is scaled by the value of $D$, if everything were diffusion controlled, all of the evolution plots versus time would be identical. Instead, at any time over the entire range probed for a multiparticle array, the average particle widths are smaller as $F^{*}$ is larger, making attachment to the edges more difficult. Also, at any given time, the lengths tend to be longer with higher $F^{*}$, evidently reflecting the fact that the matrix supersaturation is higher if the sides cannot efficiently attach solute. The effect of large $F^{*}$ on the widths is strong for both particle growth and the coarsening regimes, but perhaps surprisingly the effect of $F^{*}$ on the lengths is only notable for particles in the competitive growth and coarsening regimes.

It can be seen that the particle aspect ratios tend to increase in time for all kinetic laws examined during the particle growth and matrix depletion stage, but during the coarsening regime, the aspect ratios tend to decrease, Fig. 8. These aspect ratio trends can be rationalized through the capillary forces. Large values of weighted mean curvature, $\kappa_{\gamma}$, tend to slow facet velocity and acts as a capillary 'back force,' this behavior can be interpreted with approximate expressions for $\kappa_{\gamma}$ for the sides and ends of a high aspect $(L / W)$ ratio particle. As the energy corresponding to extension of the long ends (of width $W$ ) derives from the surface tension of the side, $\kappa_{\gamma}($ end $) \approx 2 \gamma_{\text {side }} / W$ and similarly $\kappa_{\gamma}($ side $) \approx 2 \gamma_{\text {end }} / L$. Because homogeneous nucleation will be dominated by configurations that have the lowest activation energy barrier 
and because the Wulff shape is the lowest total surface energy configuration at any volume, homogeneous nuclei will tend to have the Wulff shape. When particles become several times larger, these capillary forces become negligible and the shapes during growth are dictated largely by kinetic considerations. However, once particles enter the coarsening regime, the driving forces are from capillary forces and these back forces again become predominant. At any instance, the local matrix concentration can be considered as being equivalent to an effective value of $\gamma /$ (particle size), and growth or shrinkage of ends and sides will depend independently on the relative value of this and the aforementioned capillary 'back forces'. For particles with linear kinetics, most particles tend toward the Wulff shapes for much of their existence. For particles with non-linear kinetics, there is a complex interplay between the kinetic and energetic considerations.

It is notable that during the depletion stage the particle lengths grow almost proportionally to time as illustrated in Fig. 7. This means that during the early stage, the matrix diffusion fields are remaining nearly constant as the ends grow. This is a situation analogous to isolated dendrites growing into supersaturated matrices, and is triggered by the anisotropy in $\gamma(\hat{n})$ and in mobilities. In contrast, for the cases with $F^{*}$ small, the sides grow proportionally to $t^{1 / 2}$; in effect they are growing into depleted matrix wherein the depletion zone is increasing in proportion to the amount of growth. This behavior is approximately similar for the single particle and arrays of particles at the early stage. We remark that when the matrix diffusivities are higher than for the examples shown, the time dependences become closer to the limits mentioned; evidently the anisotropy in local growth kinetics leading to formation of narrow particles is a trigger to the formation of dendrite-like diffusion fields ahead of the rapidly growing particle end. In contrast, when the diffusivities are lower, the aspect ratios become small during growth limited by the arrival rate of solute, especially with low $F^{*}$.

The formation of steps and new facets by subdivision is physically (and algorithmically) necessary to produce the dendrite-like and other important behaviors. $\mathrm{N}$, facet formation allows a particle to change its shape and absorb solute that is distributed inhomogeneously along a facet, i.e., smaller facets can form in neighborhoods of large supersaturation and grow with increased velocity; however facets cannot be too small because of the capillary 'back forces'. Furthermore, sub-facet formation allows particles to 'grow around' other particles; thus, microstructural interlocking and impingement are directly related to material parameters and not simply to initial geometry. 
The plots of the trajectories of individual particles merit a few comments. At short times, the aspect ratios of all particles tend to increase as does the average. However, once growth becomes fully competitive, the aspect ratios tend to diminish for reasons discussed, Fig. 9. Particles of about the average size will tend not to be growing or shrinking but can be shifting toward the Wulff shape. For the nonlinear cases, particles can shrink along the ends and lose mass even though the sides are growing more slowly. In all instances, once the shrinking particles become much smaller than the critical size, the details of the difference in capillary 'back forces' for ends and side become less important and particle shapes again become kinetically determined. With low diffusivities, the shapes tend toward $A=1$, seemingly independent of the Wulff shape; however, at the final death throes, the attachment kinetics come into play again. Both from Fig. 9 and 6, it can be seen that the "ends" shrink faster than the sides, and from the snapshots in Fig. 6, it can be seen that the orientation of the long and short direction switches, such that the direction with high attachment kinetics becomes the short direction of the aspect ratio.

The data displayed in Fig. 10 show distributions of aspect ratio or of length plotted versus actual particle width, as is typical of accessible data from experiments. Such distributions depend on the relative values of $F^{*}$ and $D$, and can be interpreted based on prior discussion and the following plots that further show the sensitivity to these parameters.

Calculations were conducted at three different values of matrix diffusivity. The results, Figs. 11 and 12, show that at fixed time $t>0$, the aspect ratio of particles is an increasing function of particle width. The spread in the aspect ratios at a fixed time decreases as time increases. A comparison between the particles with strictly linear kinetic laws for all facets and the particles with parabolic/linear sides shows that while the particle length distributions are comparable, the particle widths are larger by approximately a factor of three for the linear case. As the matrix diffusivity is decreased the spread of lengths decreases. This may be because as the solute diffusion in the matrix is reduced, solute that can't join a particle side facet isn't redistributed as quickly and so has more chance to join that facet at a later time. When the diffusivity is higher, solute that may be building up near a side facet with a low mobility diffuses through the matrix to reduce chemical potential gradients. In this instance, the solute is more likely to join a particle end facet than in the case of lower matrix diffusivity.

A unified way of describing the simulation conditions tested here is to 
identify $F^{*}$. For a facet with strictly linear kinetics, $F^{*}=0$. As expected, at fixed $D$ when $F^{*}$ increases, the particle aspect ratios will increase as the velocity of the sides is lower with the same $F$ than the velocity of the ends, Figs. 11 and 12. At fixed $F^{*}>0$, as $D$ increases the particle aspect ratios will increase due to the transport of solute through the matrix away from the sides where it may build up, as is born out by examining Fig. 12. However, at large $F^{*}$, in a closed box simulation, the particle lengths will reach a threshold due to the particle impingement and interconnection of the microstructure, Figure 6 b).

The model in this paper can be used to interpret the particle growth statistics from supersaturated glass experiments with Lu and La doped glasses [29]. The aspect ratios of Lu-doped particles are initially high but at long times approach two and are near to this value during most of growth. In contrast, the aspect ratios of La-doped particles have a comparatively larger spread and average and don't converge at long times. On average, the particle widths are larger and lengths smaller for Lu-doped compared to La-doped glass. Satet et al. note that the diffusivity of $\mathrm{Si}$ and $\mathrm{N}$ in the two glasses is comparable. Therefore, the differences between $\mathrm{Lu}$ and La-doped materials could be modeled by changing $F^{*}$ so that $F_{L u}^{*}<F_{L a}^{*}$. From present results, it can be inferred that for the particles with Lu-doping that are growing with an aspect ratio of two, this value may relate more to that of the Wulff shape than of kinetic anisotropy. For the Y-doped system, Fig. 3, the statistical data indicate that behavior is intermediate between that with $\mathrm{Lu}$ and $\mathrm{La}$ doping.

Preliminary results from high resolution transmission electron microscopy (TEM) indicate that terminating planes at intergranular pockets in Y-doped silicon nitride appear faceted at a small scale while terminating planes in undoped material appear rough at the atomic scale [48]. This suggests that Y segregates to the grain/pocket interfaces, at least in part in an ordered manner, and could, thereby, increase the step nucleation energy to effectively poison growth of those planes. Z-contrast scanning TEM is providing evidence that La atoms segregate to intergranular films in silicon nitride [8] with some order at the prism face. Unpublished work at Oxford shows different distributions of $\mathrm{Lu}$ and La atoms at prism surfaces in polycrystalline silicon nitride [49]. Therefore, in the glass experiments, different re-organization routines may proceed at the prism plane interfaces with $\mathrm{Lu}$ and La adsorbed. 


\section{Conclusions}

The model described here is simple yet versatile. It is possible to test growth parameters and laws that effect the evolution of a set of two-dimensional, strictly faceted particles due to growth from depletion of solute from a supersaturated matrix and competitive growth with other particles in the set. Two kinetic laws were demonstrated with linear, $F^{*}=0$, and parabolic growth, $F^{*}>0$. For driving force less than zero and greater than $F^{*}$, the growth law is linear. For all other driving forces, the growth law is parabolic and slower than linear.

Calculations with different values of $F^{*}$ and matrix diffusivity, $D$, illustrate three trends.

1. Aspect ratios increase during the particle growth stage, to a maximum dictated by growth kinetics and particle impingement, and then decrease during coarsening by an amount that is sensitive to the nonlinearity of the growth kinetics.

2. For constant $D$, the particle aspect ratios increase with increasing $F^{*}$.

3. For constant $F^{*}$, the particle aspect ratios increase with increasing $D$, which shifts kinetics toward being attachment limited.

The numerical simulations provide several advantages. First, the need to divide microstructural evolution into various 'model' stages is artificial and unnecessary - growth kinetics evolve naturally into coarsening kinetics, and, while distinct with characteristic exponents, are part of the same model, just as they are part of the same physical processes. The numerical solutions done to date demonstrate that self-similarity of the evolving particle size distribution, which underlies scaling in the LSW kinetics, is not achieved for anisotropic coarsening with particle number reductions by a factor exceeding 10. The numerical simulations provide a framework for comparison to experimental data without the need for potentially unphysical model assumptions. However, ostensibly useful scaling laws should still apply and require investigation. Perhaps, such numerical simulations may stimulate better, localized, particle size distribution modeling.

For the simulations provided here, linear kinetics create particle shapes which tend towards the Wulff shape at low driving forces, i.e., in the coarsening regime. Depending on the magnitude of the non-linearity, non-linear 
kinetics do not produce Wulff shapes, but a distribution of shapes dictated at its extremes more by a kinetic shape than by a Wulff shape.

Comparison of statistics from simulations to those from physical experiments suggest that the observed differences between Lu and La doped $\mathrm{Si}_{3} \mathrm{~N}_{4}$ behavior could be modeled by $F_{L u}^{*}<F_{L a}^{*}$ as the matrix diffusivities are expected to be similar.

In the future, we intend to explore exponential growth laws representing island nucleation and facet area dependent growth laws representing constant defect densities. Calculations will be conducted with low supersaturations in order to compare them to the model particle in glass growth experiments. Higher supersaturation calculations will be conducted with parameters representing different $\mathrm{RE}$ additives in $\mathrm{Si}_{3} \mathrm{~N}_{4}$ in order to simulate dense ceramics.

\section{Acknowledgments}

CMB and WCC acknowledge support from NSF Grant DMR-0010062. RLS acknowledges support from EU Commission Contract G5RD-CT-2001-00586. RMC was supported by Office of Basic Energy Science, Division of Materials Sciences and Engineering of Dept. of Energy under Contract No. DE-AC0376F00098. The discussions and hospitality of Dr. Dominique Chatain and of Profs. Adrian Sutton and David Cockayne are gratefully acknowledged by WCC and CMB.

\section{References}

[1] G. Petzow, M. Herrmann: High Performance Non-Oxide Ceramics II Structure and Bonding 102 (2002) 47.

[2] P. Sajgalik, J. Dusza, M. J. Hoffmann: J. Am. Ceram. Soc. 78 (1995) 2619.

[3] P. F. Becher, E. R. Fuller, P. Angelini: J. Am. Ceram. Soc. 74 (1991) 2131.

[4] P. F. Becher, E. Y. Sun, K. P. Plucknett, K. B. Alexander, C.-H. Hsueh, H.-T. Lin, S. B. Waters, C. G. Westmoreland, E.-S. Kang, K. Hirao, M. E. Brito: J. Am. Ceram. Soc. 81 (1998) 2821. 
[5] T. Ohji, K. Hirao, S. Kanzaki: J. Am. Ceram. Soc. 78 (1995) 3125.

[6] C.-M. Wang, X. Pan, M. J. Hoffmann, R. M. Cannon, M. Rühle: J. Am. Ceram. Soc. 79 (1996) 788.

[7] M. J. Hoffmann, H. Gu, R. M. Cannon: Mater. Res. Soc. Symp. Proc. 586 (2000) 65.

[8] N. Shibata, S. J. Pennycook, T. R. Gosnell, G. S. Painter, W. A. Shelton, P. R. Becher: Nature 428 (2004) 730.

[9] Y. Goto, G. Thomas: Acta Metall. Mater. 43 (1995) 923.

[10] R. L. Satet, M. J. Hoffmann: J. Eur. Ceram. Soc. 24 (2004) 3437.

[11] L.-L. Wang, T.-Y. Tien, I.-W. Chen: J. Am. Ceram. Soc. 81 (1998) 2677.

[12] S.-J. L. Kang, S.-M. Han: Mater. Res. Bull. 20 (1995) 33.

[13] N. P. Padture: J. Am. Ceram. Soc. 77 (1994) 519.

[14] S. K. Lee, C. H. Kim: J. Am. Ceram. Soc. 77 (1994) 1955.

[15] W. J. MoberlyChan, J. J. Cao, L. C. De-Jonghe: Acta Mater. 46 (1998) 1625 .

[16] X.-F. Zhang, Q. Yang, L. C. De-Jonghe: Acta Mater. 51 (2003) 3849.

[17] H. Song, R. L. Coble: J. Am. Ceram. Soc. 73 (1990) 2086.

[18] D. S. Horn, G. L. Messing: Mater. Sci. Eng. A 195 (1995) 169.

[19] M. M. Seabaugh, E. Suvaci, B. Brahmaroutu, G. L. Messing: Interface Science 8 (2002) 257.

[20] M.-K. Kang, Y.-S. Yoo, D.-Y. Kim, N. M. Hwang: J. Am. Ceram. Soc. $83(2000) 385$.

[21] B. K. Lee, S. Y. Chung, S.-J. L. Kang: Acta Mater. 48 (2000) 1575.

[22] S. J. Bennison, M. P. Harmer: Ceram. Trans. 7 (1990) 13. 
[23] K. L. Gavrilov, S. J. Bennison, K. R. Mikeska, R. Levi-Setti: Acta Mater. 47 (1999) 4031.

[24] S.-H. Lee, D.-Y. Kim, N. M. Hwang: J. Eur. Ceram. Soc. 22 (2002) 317.

[25] B. K. Kim, S.-H. Hong, S.-H. Lee, D.-Y. Kim, N. M. Hwang: J. Am. Ceram. Soc. 86 (2003) 634.

[26] I. MacLaren, R. M. Cannon, M. A. Gülgün, R. Voytovych, N. PopescuPogrion, C. Scheu, U. Täffner, M. Rühle: J. Am. Ceram. Soc. 86 (2003) 650.

[27] C. Scott, M. Kaliszewski, C. Greskovich, L. Levinson: J. Am. Ceram. Soc. 85 (2002) 1275.

[28] M. Kramer, M. J. Hoffmann, G. Petzow: J. Am. Ceram. Soc. 76 (1993) 2778 .

[29] R. L. Satet, M. J. Hoffmann, C. M. Bishop, W. C. Carter, R. M. Cannon, A. R. Roosen: in preparation (2004).

[30] I. M. Lifshitz, V. V. Slyozov: J. Phys. Chem. Solids 19 (1961) 35.

[31] C. Wagner: Z. Elektrochem. 65 (1961) 581.

[32] A. J. Ardell: J. Eur. Ceram. Soc. 19 (1999) 2217.

[33] J. Alkemper, V. A. Snyder, N. Akaiwa, P. W. Voorhees: Phys. Rev. Lett. 82 (1999) 2725.

[34] J.-R. Lai, Y.-Y. Tien: J. Am. Ceram. Soc. 76 (1993) 91.

[35] M. Kitayama, K. Hirao, M. Toriyama, S. Kanzaki: J. Am. Ceram. Soc. 46 (1998) 6541.

[36] M. F. Yan, R. M. Cannon, H. K. Bowen, U. Chowdhry: Mater. Sci. Eng. $60(1983) 275$.

[37] D. J. Srolovitz, G. S. Grest, M. P. Anderson: Acta Metall. 33 (1985) 2233.

[38] C. V. Thompson, H. J. Frost, F. Spaepen: Acta Metall. 35 (1987) 887. 
[39] C. W. Park, D. Y. Yoon: Ceram. Trans. 118 (2000) 127.

[40] A. R. Roosen, W. C. Carter: Physica A 261 (1998) 232.

[41] J. W. Martin, R. D. Doherty, B. Cantor: Stability of Microstructures in Metallic Systems, Cambridge University Press, New York (1997).

[42] W. K. Burton, N. Cabrera, F. C. Frank: Philosophical Transactions of the Royal Society of London, Series A 243 (1951) 299.

[43] Y. J. Park, N. M. Hwang, D. Y. Yoon: Metall. Mater. Trans. A 27 (1996) 2809.

[44] G. S. Rohrer, C. L. Rohrer, W. W. Mullins: J. Am. Ceram. Soc. 85 (2002) 675 .

[45] A. R. Roosen, J. E. Taylor: Materials Research Society Proceedings 237 (1992) 25.

[46] W. C. Carter, A. R. Roosen, J. W. Cahn, J. E. Taylor: Acta. Metall. 43 (1995) 4309.

[47] J. E. Taylor: Acta Mater. 40 (1992) 1475.

[48] M. Döblinger: unpublished research (2004).

[49] G. B. Winkelman, C. Dwyer, T. S. Hudson, D. Nguyen-Manh, M. Döblinger, R. L. Satet, M. J. Hoffmann, D. J. H. Cockayne: submitted to Phys. Rev. Lett. (2004).

Correspondence address: Dr. Catherine M. Bishop

Department of Materials, Parks Road, Oxford, OX2 3PH, UK

Tel: +44 1865273672

Fax: +44 1865283333 